\title{
Lowering blood pressure in black African patients
}

Dual therapies combining a calcium-channel blocker with an angiotensin-converting enzyme (ACE) inhibitor or thiazide diuretic are more effective at lowering blood pressure in black African patients than the combination of an ACE inhibitor and a thiazide diuretic, according to results presented at ACC. 19.

Dual therapies are needed in many patients to control blood pressure effectively; however, recommendations for combination therapies in black patients differ across three guidelines in the USA and the latest European guidelines. "We decided to do this research because before now no large randomized controlled trials have compared the efficacy of contemporary combination therapies among any black populations, in spite of the high burden of hypertension and its complications in this population," explains Dike Ojji, corresponding author.

In this trial, 728 black patients with uncontrolled hypertension from six countries in sub-Saharan Africa were randomly assigned to receive amlodipine (a calcium-channel blocker) and hydrochlorothiazide (a thiazide diuretic), amlodipine and perindopril (an ACE inhibitor) or perindopril and hydrochlorothiazide.

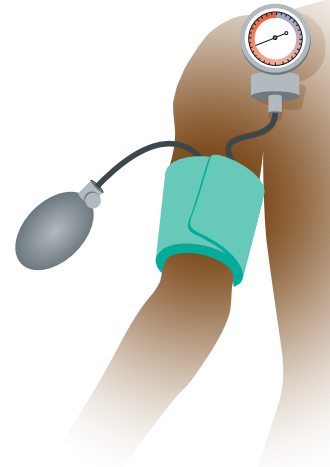

The primary end point was change in 24-h ambulatory systolic blood pressure between baseline and 6 months.

Patients who received either of the combinations containing amlodipine had a larger reduction in blood pressure than those patients who received perindopril and hydrochlorothiazide. The efficacy of calcium-channel blockers as monotherapy in black patients is well established, and the results from this study suggest that a calcium-channel blocker might also be the vital component in dual therapies.

"This study emphasizes the need to establish optimal antihypertensive combination therapies by ethnicity," says Ojji. "It would also be interesting to carry out the same project in African Americans and compare results as it is difficult to extrapolate our work to this population because of gene-environment interaction and genetic admixture."

Isobel Leake

ORIGINAL ARTICLE Ojji, D. B. et al. Compariso

of dual therapies for lowering blood pressure in

Black Africans. N. Engl.J. Med. https://doi.org/

10.1056/NEJMoa1901113 (2019)

\section{Cholesterol efflux drives stem cell expansion in hypercholesterolaemia}

Hypercholesterolaemia induces the expansion of haematopoietic stem and progenitor cells (HSPCs), resulting in increased leukocyte numbers, which contribute to atherosclerotic cardiovascular disease. However, the mechanisms linking hypercholesterolaemia and HSPC expansion are unclear. Longhou Fang and colleagues describe a new mechanism for HSPC emergence in embryogenesis that also regulates adult haematopoiesis in hypercholesterolaemic conditions.

The investigators show that during embryogenesis in zebrafish, apolipoprotein A-I binding protein 2 (Aibp2; also known as Yjefn3) - a pro-haematopoietic cue derived from the somite that binds to HDL, which augments cholesterol efflux - increases HSPC specification from the haemogenic endothelium through the activation of sterol regulatory elementbinding protein 2 (Srebp2), the master regulator of cholesterol biosynthesis.
Aibp2-mediated cholesterol efflux activates endothelial Srebp2, which in turn transactivates the Notch pathway, promoting HSPC emergence.

In Ldlr-knockout mice fed a highcholesterol diet, inhibition of SREBP2 repressed the hypercholesterolaemiainduced HSPC expansion in the bone marrow, suggesting that SREBP2-

SREBP2regulated Notch signalling contributes to HSPC expansion during hypercholesterolaemia regulated Notch signalling contributes to HSPC expansion during hypercholesterolaemia in adults. Previous studies in mice showed that SREBP2 inhibition reduces atherosclerotic burden. "We speculate that SREBP2 suppression protects against cardiovascular disease in part by targeting aberrant haematopoiesis," explains Fang.

Finally, the investigators confirmed the relevance of their findings in humans, showing that increased SREBP 2 and Notch signalling activation was associated with HSPC expansion in patients with hypercholesterolaemia.

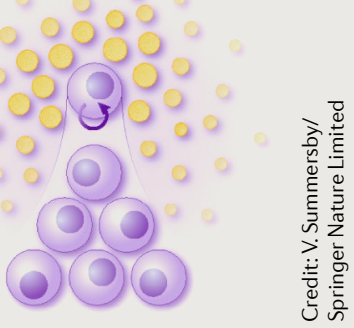

The research team plans to continue exploring the role of cholesterol metabolism in haematopoiesis. "Generation of autologous, bona fide HSPCs is essential for regenerative medicine, and our findings may bring us one step closer to this ultimate goal," highlights Fang. "Our studies also provide new therapeutic targets for immunological and haematological diseases, as well as for cardiovascular disease." Irene Fernández-Ruiz

ORIGINAL ARTICLE Gu, Q. et al. AlBP-mediated cholesterol efflux instructs hematopoietic stem and progenitor cell fate. Science 363, 1085-1088 (2019) FURTHER READING Rahman, M. S. et al. Effects of dyslipidaemia on monocyte production and function in cardiovascular disease. Nat. Rev. Cardiol. 14, 387-400 (2017) 